\title{
Solving second order delay differential equations by direct extended two and three point implicit one-step block method
}

\begin{abstract}
The purpose of this paper is to solve directly the second order delay differential equations (DDEs) using The extended two and extended three point implicit one-step block methods using constant step size. The formulae for the extended two and extended three point implicit one-step block methods will be derived using Lagrange interpolation polynomial. The stability polynomials for the methods are obtained and their regions of absolute stability are discussed. The efficiency of the proposed method is supported by some numerical results.
\end{abstract}

Keyword: Direct extended block method; Retarded delay differential equations 